\title{
Sequentially Congruent Partitions and Related Bijections
}

\author{
Maxwell Schneider and Robert Schneider
}

In honor of George E. Andrews on his 80th birthday

\begin{abstract}
We study a curious class of partitions, the parts of which obey an exceedingly strict congruence condition we refer to as "sequential congruence": the $m$ th part is congruent to the $(m+1)$ th part modulo $m$, with the smallest part congruent to zero modulo the length of the partition. It turns out these obscure-seeming objects are embedded in a natural way in partition theory. We show that sequentially congruent partitions with largest part $n$ are in bijection with the partitions of $n$. Moreover, we show sequentially congruent partitions induce a bijection between partitions of $n$ and partitions of length $n$ whose parts obey a strict "frequency congruence" condition - the frequency (or multiplicity) of each part is divisible by that part - and prove families of similar bijections, connecting with G. E. Andrews's theory of partition ideals.
\end{abstract}

Mathematics Subject Classification (2010). Primary 05A17; Secondary $11 \mathrm{P} 84$.

Keywords. Partitions, $q$-series, generating function, partition ideal.

\section{Introduction}

Here we consider a somewhat exotic subset of integer partitions, which turns out to be naturally embedded in partition theory.

Let $\mathcal{P}$ denote the set of partitions, with elements $\lambda=\left(\lambda_{1}, \lambda_{2}, \ldots, \lambda_{r}\right)$, $\lambda_{1} \geq \lambda_{2} \geq \ldots \geq \lambda_{r} \geq 1$, including the empty partition $\emptyset$. Alternatively, following Andrews [4, Fine [8] and other authors, one sometimes writes $\lambda=$ $\left(\begin{array}{llll}1^{m_{1}} & 2^{m_{2}} & 3^{m_{3}} & \ldots\end{array}\right)$ with $m_{i}=m_{i}(\lambda)$ the frequency (or multiplicity) of $i \in \mathbb{N}$ as a part of $\lambda$, setting $m_{i}(\emptyset)=0$ for all $i$. Furthermore, for a given partition $\lambda$, let $|\lambda|$ denote its size (sum of the parts) and $\ell(\lambda):=r$ denote its length (number of parts), with the conventions $|\emptyset|:=0, \ell(\emptyset):=0$.

We define the set $\mathcal{S} \subset \mathcal{P}$ of sequentially congruent partitions as follows. 
Definition 1.1. We define a partition $\lambda$ to be sequentially congruent if the following congruences between the parts are all satisfied:

$$
\begin{gathered}
\lambda_{1} \equiv \lambda_{2}(\bmod 1), \lambda_{2} \equiv \lambda_{3}(\bmod 2), \lambda_{3} \equiv \lambda_{4}(\bmod 3), \ldots, \\
\lambda_{r-1} \equiv \lambda_{r}(\bmod r-1),
\end{gathered}
$$

and for the smallest part, $\lambda_{r} \equiv 0(\bmod r)$.

For example, the partition $(20,17,15,9,5)$ is sequentially congruent, because $20 \equiv 17(\bmod 1)$ trivially, $17 \equiv 15(\bmod 2), 15 \equiv 9(\bmod 3), 9 \equiv$ $5(\bmod 4)$, and finally $5 \equiv 0(\bmod 5)$. On the other hand, $(21,18,16,10,6)$ is not sequentially congruent, for while the first four congruences still hold, clearly $6 \not \equiv 0(\bmod 5)$. Note that increasing the largest part $\lambda_{1}$ of any $\lambda \in \mathcal{S}$ yields another partition in $\mathcal{S}$, as does adding or subtracting a fixed integer multiple of the length $r$ to all its parts, so long as the resulting parts are still positive.

No doubt, this strict congruence restriction on the parts hardly appears natural. However, it turns out sequentially congruent partitions are in oneto-one correspondence with the entire set $\mathcal{P}$.

\section{Bijections Between $\mathcal{S}$ and $\mathcal{P}$}

Let $\mathcal{P}_{n}$ denote the set of partitions of $n$, as usual let $p(n)=\# \mathcal{P}_{n}$ (with $\# Q$ the cardinality of a set $Q$ ), and let $\mathcal{S}_{\lg =n}$ denote sequentially congruent partitions $\lambda^{\prime}$ whose largest part $\lambda_{1}^{\prime}$ equals $n$.

Theorem 2.1. There exists a bijection $\pi$ between the set $\mathcal{P}$ and the set $\mathcal{S}$ such that

Moreover, we have

$$
\pi\left(\mathcal{P}_{n}\right)=\mathcal{S}_{\mathrm{lg}=n}
$$

$$
\# \mathcal{S}_{\lg =n}=p(n) \text {. }
$$

Proof. We prove the theorem directly by construction.

For partition $\lambda=\left(\lambda_{1}, \lambda_{2}, \ldots, \lambda_{i}, \ldots, \lambda_{r}\right)$, one constructs a sequentially congruent dual

$$
\lambda^{\prime}=\left(\lambda_{1}^{\prime}, \lambda_{2}^{\prime}, \ldots, \lambda_{i}^{\prime}, \ldots, \lambda_{r}^{\prime}\right)
$$

by taking the parts equal to

$$
\lambda_{i}^{\prime}=i \lambda_{i}+\sum_{j=i+1}^{r} \lambda_{j} .
$$

Note that $\lambda_{r}^{\prime} \equiv 0(\bmod r)$ as $\sum_{j=r+1}^{r}$ is empty; the other congruences between successive parts of $\lambda^{\prime}$ are also immediate from equation (2.1).

Let us take

$$
\pi: \mathcal{P} \rightarrow \mathcal{S}
$$

to be the map defined by this construction, with $\lambda^{\prime}=\pi(\lambda)$. The above argument establishes, in fact, that we have more strongly $\pi: \mathcal{P}_{n} \rightarrow \mathcal{S}_{\text {lg }=n}$. 
Conversely, given a sequentially congruent partition $\lambda^{\prime}$, one can recover the dual partition $\lambda$ by working from right-to-left. Begin by computing the smallest part

$$
\lambda_{r}=\frac{\lambda_{r}^{\prime}}{r}
$$

then compute $\lambda_{r-1}, \lambda_{r-2}, \ldots, \lambda_{1}$ in this order by taking

$$
\lambda_{i}=\frac{1}{i}\left(\lambda_{i}^{\prime}-\sum_{j=i+1}^{r} \lambda_{j}\right)
$$

We define the inverse map $\pi^{-1}$ from the algorithm in (2.2) and (2.3), i.e., $\pi^{-1}\left(\lambda^{\prime}\right)=\lambda$ :

$$
\pi^{-1}: \mathcal{S} \rightarrow \mathcal{P}
$$

Noting that the uniqueness of $\lambda$ implies the uniqueness of $\lambda^{\prime}$, and vice versa, the bijection between $\mathcal{S}$ and $\mathcal{P}$ follows from this two-way construction.

Furthermore, since $\lambda_{1}^{\prime}=|\lambda|$, then every partition $\lambda$ of $n$ corresponds to a sequentially congruent partition $\lambda^{\prime}$ with largest part $n$, and vice versa.

The sets $\mathcal{P}$ and $\mathcal{S}$ enjoy another interrelation that can be used to compute the coefficients of infinite products. Now, it is a rewriting of Equation 22.16 in Fine [8] that for a function $f: \mathbb{N} \rightarrow \mathbb{C}$ and $q \in \mathbb{C}$ with $f, q$ chosen such that the product converges absolutely, we have

$$
\prod_{n=1}^{\infty}\left(1-f(n) q^{n}\right)^{-1}=\sum_{\lambda \in \mathcal{P}} q^{|\lambda|} \prod_{i \geq 1} f(i)^{m_{i}}
$$

where $m_{i}=m_{i}(\lambda)$ is the frequency of $i$ as a part of $\lambda$, and the sum on the right is taken over all partitions $\lambda$. Of course the canonical case would be, for $|q|<1$, the identity

$$
\prod_{n=1}^{\infty}\left(1-x q^{n}\right)^{-1}=\sum_{\lambda \in \mathcal{P}} x^{\ell(\lambda)} q^{|\lambda|}
$$

which enjoys many beautiful $q$-series representations (see [4, 6, 8, )

It follows from an extension of (2.5) in [10] that the product on the left side of (2.5) can also be expressed as a sum over sequentially congruent partitions.

Let $\lg (\lambda)=\lambda_{1}$ denote the largest part of partition $\lambda$, and set $\lambda_{k}=0$ if $k>\ell(\lambda)$.

Theorem 2.2. For $f: \mathbb{N} \rightarrow \mathbb{C}, q \in \mathbb{C}$ such that the product converges absolutely, we have

$$
\prod_{n=1}^{\infty}\left(1-f(n) q^{n}\right)^{-1}=\sum_{\lambda \in \mathcal{S}} q^{\lg (\lambda)} \prod_{i \geq 1} f(i)^{\left(\lambda_{i}-\lambda_{i+1}\right) / i} .
$$


Proof of Theorem 2.2. For $j=1,2,3, \ldots$, let $\mathcal{P}_{T_{j}}$ denote partitions whose parts are all in some subset $T_{j} \subseteq \mathbb{N}$, with $\emptyset \in \mathcal{P}_{T_{j}}$ for all $j$, and define $f_{j}: T_{j} \rightarrow \mathbb{C}$. To prove Theorem 2.2 , we begin by recalling Corollary 2.9 of [10] in the case that " \pm " signs are set to minus:

$$
\prod_{j=1}^{n} \prod_{k_{j} \in T_{j}}\left(1-f_{j}\left(k_{j}\right) q^{k_{j}}\right)^{-1}=\sum_{k=0}^{\infty} c_{k} q^{k},
$$

with the coefficients $c_{k}$ given by the somewhat unwieldy $(n-1)$-tuple sum

$$
\begin{aligned}
c_{k}= & \sum_{k_{2}=0}^{k} \sum_{k_{3}=0}^{k_{2}} \cdots \sum_{k_{n}=0}^{k_{n-1}}\left(\sum_{\substack{\lambda \vdash k_{n} \\
\lambda \in \mathcal{P}_{T_{n}}}} \prod_{\lambda_{i} \in \lambda} f_{n}\left(\lambda_{i}\right)\right)\left(\sum_{\substack{\lambda \vdash\left(k_{n-1}-k_{n}\right) \\
\lambda \in \mathcal{P}_{T_{n-1}}}} \prod_{\lambda_{i} \in \lambda} f_{n-1}\left(\lambda_{i}\right)\right) \\
& \times\left(\sum_{\substack{\lambda \vdash\left(k_{n-2}-k_{n-1}\right) \\
\lambda \in \mathcal{P}_{T_{n-2}}}} \prod_{\lambda_{i} \in \lambda} f_{n-2}\left(\lambda_{i}\right)\right) \\
& \left(\sum_{\substack{\lambda \vdash\left(k-k_{2}\right) \\
\lambda \in \mathcal{P}_{T_{1}}}} \prod_{\lambda_{i} \in \lambda} f_{1}\left(\lambda_{i}\right)\right),
\end{aligned}
$$

where " $\lambda \vdash r$ " indicates $\lambda$ is a partition of $r$ and the interior products are taken over the parts $\lambda_{i}$ of each $\lambda$, which identity can be proved from (2.5) by repeated application of the Cauchy product formula.

Now, for every $j \in \mathbb{N}$ take $T_{j}=\{j\}$ and fix $f_{j}=f$. In this case, $\lambda \in \mathcal{P}_{T_{j}}$ means if $\lambda \neq \emptyset$ that $\lambda=(j, j, \ldots, j)$, so we must have $j \mid\left(k_{j}-k_{j+1}\right)$ in any nonempty partition sum on the right side above. Then every summand comprising $c_{k}$ vanishes unless all the $k_{i} \leq k$ are parts of a sequentially congruent partition having length $\leq n$ : each sum over partitions is empty (i.e., equal to zero) if $j$ does not divide $k_{j}-k_{j+1}$; is equal to 1 if $k_{j}-k_{j+1}=0$ as then $\lambda=\emptyset$ and $\prod_{\lambda_{i} \in \emptyset}$ is an empty product; or else has one term $f(j)^{m_{j}}=f(j)^{\left(k_{j}-k_{j+1}\right) / j}$ as there is exactly one $\lambda=(j, j, \ldots, j)$ with $|\lambda|=m_{j} j=k_{j}-k_{j+1}>0$. Finally, let $n \rightarrow \infty$ so this argument encompasses partitions in $\mathcal{S}$ of unrestricted length.

Remark 2.3. We note that setting $f=1$, then comparing equation (2.5) to Theorem 2.2 gives another proof of Theorem 2.1 the sets $\mathcal{S}_{\mathrm{lg}=n}$ and $\mathcal{P}_{n}$ (and thus, the sets $\mathcal{S}$ and $\mathcal{P}$ ) have the same product generating function.

Remark 2.4. If we instead take every \pm equal to plus in Corollary 2.9 of [10, similar arguments reveal there is also a bijection between partitions into distinct parts and the subset of $\mathcal{S}$ containing partitions into parts with differences $\lambda_{i}-\lambda_{i+1}=i$ exactly.

\section{Cyclic Sequentially Congruent Maps}

Comparing Theorem 2.2 with (2.5) above, we have two formally differentlooking decompositions of the coefficients of $\prod_{n \geq 1}\left(1-f(n) q^{n}\right)^{-1}$ as sums over 
partitions of the form $\sum_{\lambda \in \mathcal{P}_{n}}$ and $\sum_{\lambda \in \mathcal{S}_{\text {lg }=n}}$, yet one observes the summands in each case consist of the same terms in different orders. Then one wonders: precisely which partition $\gamma \in \mathcal{P}_{n}$ is such that

$$
\prod_{i \geq 1} f(i)^{\left(\phi_{i}-\phi_{i+1}\right) / i}=\prod_{j \geq 1} f(j)^{m_{j}(\gamma)}
$$

for a given $\phi \in \mathcal{S}_{\mathrm{lg}=n}$ ? One observes that $\gamma$ is generally not the same partition $\lambda=\pi^{-1}(\phi)$ as in (2.4).

Evidently the set $\mathcal{S}$ enjoys a second map to $\mathcal{P}$ (apart from $\pi^{-1}$ ). Let

$$
\sigma: \mathcal{S} \rightarrow \mathcal{P}
$$

denote this map. We can write $\sigma$ down by comparing the forms of the products in (3.1):

$$
\sigma(\phi):=\left(1^{\phi_{1}-\phi_{2}} 2^{\left(\phi_{2}-\phi_{3}\right) / 2} 3^{\left(\phi_{3}-\phi_{4}\right) / 3} \ldots\right)=\gamma \in \mathcal{P}_{n},
$$

where $\phi \in \mathcal{S}_{\lg =n}$ as above. For example, $\sigma(5,3,3)=\left(1^{5-3} 2^{(3-3) / 2} 3^{(3-0) / 3}\right)=$ $(3,1,1)$.

Under this map we have $\sigma\left(\mathcal{S}_{\mathrm{lg}=n}\right)=\mathcal{P}_{n}$, thus the composite map is

$$
\sigma \circ \pi: \mathcal{P}_{n} \rightarrow \mathcal{P}_{n},
$$

and, similarly, we have the map $\pi \circ \sigma: \mathcal{S}_{\mathrm{lg}=n} \rightarrow \mathcal{S}_{\mathrm{lg}=n}$.

A natural question to ask is: what kind of permutation structure arises as we alternately compose $\pi, \sigma$, that is, what if we apply $\sigma \circ \pi \circ \sigma \circ \pi \circ \cdots \circ \sigma \circ \pi$ to a partition of $n$ ? For a concrete example, let's check by repeatedly applying $\sigma \circ \pi \circ \cdots \circ \sigma \circ \pi$ to the partitions of $n=4$ :

$$
\begin{gathered}
(4) \stackrel{\pi}{\longmapsto}(4) \stackrel{\sigma}{\longmapsto}(1,1,1,1) \stackrel{\pi}{\longmapsto}(4,4,4,4) \stackrel{\sigma}{\longmapsto}(4), \\
(3,1) \stackrel{\pi}{\longmapsto}(4,2) \stackrel{\sigma}{\longmapsto}(2,1,1) \stackrel{\pi}{\longmapsto}(4,3,3) \stackrel{\sigma}{\longmapsto}(3,1), \\
(2,2) \stackrel{\pi}{\longmapsto}(4,4) \stackrel{\sigma}{\longmapsto}(2,2), \\
(2,1,1) \stackrel{\pi}{\longmapsto}(4,3,3) \stackrel{\sigma}{\longmapsto}(3,1) \stackrel{\pi}{\longmapsto}(4,2) \stackrel{\sigma}{\longmapsto}(2,1,1), \\
(1,1,1,1) \stackrel{\pi}{\longmapsto}(4,4,4,4) \stackrel{\sigma}{\longmapsto}(4) \stackrel{\pi}{\longmapsto}(4) \stackrel{\sigma}{\longmapsto}(1,1,1,1) .
\end{gathered}
$$

There appears to be cyclic behavior of order 1 or 2 ; also evident is the following fact.

Theorem 3.1. The composite map $\sigma \circ \pi: \mathcal{P}_{n} \rightarrow \mathcal{P}_{n}$ takes partitions to their conjugates.

Proof. If we write

$$
\lambda=\left(a_{1}^{m_{a_{1}}} a_{2}^{m_{a_{2}}} a_{3}^{m_{a_{3}}} \ldots a_{r}^{m_{a_{r}}}\right), a_{1}>a_{2}>\ldots>a_{r} \geq 1,
$$

then we can compute the parts and frequencies of the conjugate partition

$$
\lambda^{*}=\left(b_{1}^{m_{b_{1}}} b_{2}^{m_{b_{2}}} b_{3}^{m_{b_{3}}} \ldots b_{s}^{m_{b_{s}}}\right), b_{1}>b_{2}>\ldots>b_{s} \geq 1,
$$

directly from the parts and frequencies of $\lambda$ by comparing the Ferrers-Young diagrams of $\lambda, \lambda^{*}$. The conjugate partition $\lambda^{*}$ has largest part $b_{1}$ given by

$$
b_{1}=\ell(\lambda)=m_{a_{1}}+m_{a_{2}}+\ldots+m_{a_{r}} \text {, with } m_{b_{1}}\left(\lambda^{*}\right)=a_{r},
$$


and for $1<i \leq s$, the parts and their frequencies are given by

$$
b_{i}=m_{a_{1}}+m_{a_{2}}+\ldots+m_{a_{r-i+1}}, \quad m_{b_{i}}\left(\lambda^{*}\right)=a_{r-i+1}-a_{r-i+2} .
$$

Moreover, we have that $s=r$. The theorem results from using the definitions of the maps $\pi$ and $\sigma$, keeping track of the parts in the transformation $\lambda \mapsto$ $(\sigma \circ \pi)(\lambda)$, then comparing the parts of $(\sigma \circ \pi)(\lambda)$ with the parts of $\lambda^{*}$ in (3.2) and (3.3) above to see they are the same.

The preceding considerations also make explicit our observation above about cyclic orders.

Corollary 3.2. We have that $(\sigma \circ \pi)(\lambda)=\lambda$ when $\lambda$ is self-conjugate, and $(\sigma \circ \pi)^{2}(\lambda)=\lambda$ holds for all $\lambda \in \mathcal{P}$. Likewise, for $\phi$ sequentially congruent it is the case that $(\pi \circ \sigma)(\phi)=\phi$ when $\sigma(\phi)$ is self-conjugate, and $(\pi \circ \sigma)^{2}(\phi)=\phi$ holds for all $\phi \in \mathcal{S}$.

Remark 3.3. Interestingly, the map $\pi \circ \sigma: \mathcal{S}_{\lg =n} \rightarrow \mathcal{S}_{\lg =n}$ defines a duality analogous to conjugation in $\mathcal{P}_{n}$, that instead connects partitions $\phi$ and $(\pi \circ$ $\sigma)(\phi)$ in $\mathcal{S}_{\mathrm{lg}=n}$. For instance, from the above examples, it is the case in $\mathcal{P}_{4}$ that $(2,1,1)$ and $(3,1)=(\sigma \circ \pi)(2,1,1)$ are conjugates, while on the same row, $(4,3,3)$ and $(4,2)=(\pi \circ \sigma)(4,3,3)$ are paired under this new, analogous duality in $\mathcal{S}_{\mathrm{lg}=4}$.

\section{Frequency Congruent Partitions and Infinite Families of Bijections}

The conjugates of sequentially congruent partitions are themselves interesting combinatorial objects.

Theorem 4.1. A sequentially congruent partition $\phi$ is mapped by conjugation to a partition $\phi^{*}$ whose frequencies $m_{i}=m_{i}\left(\phi^{*}\right)$ obey the congruence condition

$$
m_{i} \equiv 0(\bmod i) .
$$

Conversely, any partition with parts obeying this congruence condition has a sequentially congruent partition as its conjugate.

Proof. The theorem is immediate by conjugation of the relevant Young diagrams.

Let us codify the objects highlighted in the preceding theorem.

Definition 4.2. We define a partition to be frequency congruent if it has the property that each part divides its frequency 1 .

Then Theorem 4.1 implies the following result.

Corollary 4.3. Frequency congruent partitions of length $n$ are in bijection with the partitions of $n$, viz.

$$
\#\left\{\lambda \in \mathcal{P}: \ell(\lambda)=n, i \mid m_{i}(\lambda)\right\}=p(n) .
$$

${ }^{1}$ As in Theorem 4.1 
Proof. This statement follows from Theorem 4.1 together with Theorem 2.1. For a combinatorial proof, take any partition $\lambda=\left(1^{m_{1}} 2^{m_{2}} 3^{m_{3}} \ldots i^{m_{i}} \ldots\right)$ of $n$, and multiply each $m_{i}$ by $i$ to yield a frequency congruent partition $\left(1^{m_{1}} 2^{2 m_{2}} 3^{3 m_{3}} \ldots i^{i m_{i}} \ldots\right)$ with length $m_{1}+2 m_{2}+3 m_{3}+\ldots=|\lambda|=n$. Conversely, by the same principle, divide the frequency of each part of a length- $n$ frequency congruent partition by the part itself for a partition of $n$.

Alternatively, we can prove the bijection using generating functions. For $|x|<1,|q|<1$, consider the following identities in light of (2.5) and (2.6):

$$
\begin{aligned}
& \prod_{n=1}^{\infty} \frac{1}{1-x^{n} q^{n^{2}}} \\
& =\left(1+x^{1} q^{1}+x^{2} q^{1+1}+x^{3} q^{1+1+1}+\ldots\right) \\
& \times\left(1+x^{2} q^{2+2}+x^{4} q^{2+2+2+2}+x^{6} q^{2+2+2+2+2+2}+\ldots\right) \\
& \times\left(1+x^{3} q^{3+3+3}+x^{6} q^{3+3+3+3+3+3}+x^{9} q^{3+3+3+3+3+3+3+3+3}+\ldots\right) \times \cdots \\
& =\sum_{\substack{\lambda \in \mathcal{P} \\
i \mid m_{i}(\lambda)}} x^{\ell(\lambda)} q^{|\lambda|}=\sum_{n=0}^{\infty} x^{n} \sum_{\substack{\ell(\lambda)=n \\
i \mid m_{i}(\lambda)}} q^{|\lambda|},
\end{aligned}
$$

where the final two (absolutely convergent) sums are taken over frequency congruent partitions.

To count the number of frequency congruent partitions of length $n$, let $q \rightarrow 1$ from within the unit the circle in the right-most series above, noting in the limit we still have convergence since $|x|<1$. Then by comparison with the product side of the generating function, the resulting coefficient of $x^{n}$ is equal to $p(n)$ by Euler's identity (see [4]).

Remark 4.4. We note that the generating function proof above provides (by conjugation) another proof that $\# \mathcal{S}_{\mathrm{lg}=n}=p(n)$.

Indeed, the steps of the preceding proof suggest a highly general frequency congruence phenomenon yielding infinite families of partition bijections.

As before, let $\mathcal{P}_{T} \subseteq \mathcal{P}$ be the set of partitions (including $\emptyset$ ) with parts from $T=\left\{t_{1}, t_{2}, t_{3}, \ldots\right\} \subseteq \mathbb{N}$; we allow $\mathcal{P}_{T}$ to also denote partitions with parts from a sequence $T$ of natural numbers if they are distinct. Let $p_{T}(n)$ denote the number of partitions of $n \geq 0$ in $\mathcal{P}_{T}$. Moreover, for a sequence $S=\left(s_{1}, s_{2}, s_{3}, \ldots\right)$ of natural numbers, define

$$
\mathcal{P}_{T}(S):=\left\{\lambda \in \mathcal{P}_{T}: s_{i} \mid m_{t_{i}}\right\},
$$

and let $\mathcal{P}_{T}(S, n)$ denote partitions in $\mathcal{P}_{T}(S)$ of length $n$. Thus $\mathcal{P}_{\mathbb{N}}((1,1,1, \ldots))$ $=\mathcal{P}$ and $\# \mathcal{P}_{\mathbb{N}}((1,1,1, \ldots), n)=p(n)$. Then we have the following.

Theorem 4.5. Let $|x|<1,|q|<1$. For a sequence $A=\left(a_{1}, a_{2}, a_{3}, \ldots\right)$ of natural numbers and subset $B=\left\{b_{1}, b_{2}, b_{3}, \ldots\right\} \subseteq \mathbb{N}$, we have

$$
\prod_{n=1}^{\infty} \frac{1}{1-x^{a_{n}} q^{a_{n} b_{n}}}=\sum_{\lambda \in \mathcal{P}_{B}(A)} x^{\ell(\lambda)} q^{|\lambda|}=\sum_{n=0}^{\infty} x^{n} \sum_{\lambda \in \mathcal{P}_{B}(A, n)} q^{|\lambda|} .
$$


If the $a_{i} \in A$ are distinct then the sets $\mathcal{P}_{A}$ and $\mathcal{P}_{B}(A)$ are in bijection, and

$$
\# \mathcal{P}_{B}(A, n)=p_{A}(n) \text {. }
$$

We note that equation (2.6) represents the case $a_{i}=1, b_{i}=i$, and the generating function in the proof of Corollary 4.3 is the case $a_{i}=b_{i}=i$.

Proof. For the first identity, much as in the proof of Corollary 4.3, for $|x|<$ $1,|q|<1$, rewrite the infinite product on the left side of Theorem 4.5 as a product of geometric series:

$$
\prod_{n=1}^{\infty}\left(1+x^{a_{n}} q^{b_{n}+b_{n}+\ldots+b_{n}}+x^{2 a_{n}} q^{b_{n}+\ldots+b_{n}}+x^{3 a_{n}} q^{b_{n}+\ldots+b_{n}}+\ldots\right)
$$

where in each term $x^{i a_{n}} q^{b_{n}+\ldots+b_{n}}$ there are $i a_{n}$ repetitions of $b_{n}$ in the exponent of $q$. Expanding the product immediately gives the first equality, and collecting coefficients of $x^{n}$ gives the right-most equality.

To prove the second identity in the theorem, just as in the proof of Corollary 4.3, let $q \rightarrow 1$ from within the unit circle in the right-most summation of the first identity. But if the $a_{i}$ are distinct the infinite product becomes

$$
\prod_{n=1}^{\infty} \frac{1}{1-x^{a_{n}}}=\prod_{n \in A} \frac{1}{1-x^{n}}=\sum_{n=0}^{\infty} p_{A}(n) x^{n} .
$$

Equating coefficients of $x^{n}$ completes the proof.

One can also prove the second identity by mapping every partition $\left(a_{1}^{m_{a_{1}}} a_{2}^{m_{a_{2}}} a_{3}^{m_{a_{3}}} \ldots\right) \in \mathcal{P}_{A}$ of size $n$ (noting these $a_{i}$ are not necessarily in increasing order $)$ to partition $\left(b_{1}^{a_{1} m_{a_{1}}} b_{2}^{a_{2} m_{a_{2}}} b_{3}^{a_{3} m_{a_{3}}} \ldots\right) \in \mathcal{P}_{B}(A, n)$ and, conversely, mapping each $\left(b_{1}^{a_{1} n_{1}} b_{2}^{a_{2} n_{2}} b_{3}^{a_{3} n_{3}} \ldots\right) \in \mathcal{P}_{B}(A, n)$ to $\left(a_{1}^{n_{1}} a_{2}^{n_{2}} a_{3}^{n_{3}} \ldots\right) 2$

Observe that in the above notation, frequency congruent partitions represent the set $\mathcal{P}_{\mathbb{N}}((1,2,3,4, \ldots))$. Recalling that the conjugates of frequency congruent partitions are sequentially congruent, then the set $\mathcal{S}_{B}(A)$ of conjugates of partitions in $\mathcal{P}_{B}(A)$ is evidently an analog of the set $\mathcal{S}$. For example, for $B=\mathbb{N}$ and sequence $A$, the conjugates of the set of partitions $\mathcal{P}_{\mathbb{N}}(A)$ such that $a_{i}$ divides $m_{i}$ have a nice sequential congruence property:

$$
\mathcal{S}_{\mathbb{N}}(A)=\left\{\lambda \in \mathcal{P}: \lambda_{i} \equiv \lambda_{i+1}\left(\bmod a_{i}\right)\right\}
$$

We conjecture there are bijective maps in this extended regime analogous to those in Sections 2 and 3 above; however, they alternate between $\mathcal{P}_{A}$ and $\mathcal{S}_{B}(A)$ under composition instead of between $\mathcal{P}$ and $\mathcal{S}$.

Remark 4.6. For $T \subseteq \mathbb{N}$ and $f, g: T \rightarrow \mathbb{C}$, two-variable generating functions of the general form $\prod_{n \in T}\left(1-x^{f(n)} q^{g(n)}\right)^{-1}$ used in this section are flexible analytic and combinatorial objects (see [4, 8]). We note if $0<x<e^{-1},|q|<$

\footnotetext{
${ }^{2}$ There is a resemblance here to maps generating other classes of partitions with nontrivial weightings on the frequencies, e.g. see [1, 5, 7, regarding identities of Capparelli and Primc.
} 
$1,1 \notin T$, taking $f(n)=\log n$ and letting $q \rightarrow 1$ as we did above yields a class of "partition zeta functions" studied in [9, 10]:

$$
\lim _{q \rightarrow 1} \prod_{n \in T}\left(1-x^{\log n} q^{g(n)}\right)^{-1}=\prod_{n \in T}\left(1-n^{\log x}\right)^{-1}=\sum_{\lambda \in \mathcal{P}_{T}} N(\lambda)^{-s},
$$

where $s:=-\log x$, thus $s>1$ for convergence, and $N(\lambda):=\prod_{\lambda_{i} \in \lambda} \lambda_{i}$. (By the same token, one may rewrite the Riemann zeta function as $\zeta(s)=$ $\zeta(-\log x)=\sum_{n=1}^{\infty} x^{\log n}$.)

\section{Further Thoughts: Partition Ideals}

In a series of papers in the 1970s (e.g. see [2, 3]), G. E. Andrews developed a theory of partition ideals which uses ideas from lattice theory to unify and extend many classical results on generating functions and partition bijections, summarized in Chapter 8 of [4].

Definition 5.1. A partition ideal is a subset $\mathcal{C} \subseteq \mathcal{P}$ with the property that if any parts are deleted from a partition in $\mathcal{C}$, the resulting partition is an element of $\mathcal{C}$ as well.

Remark 5.2. We note Andrews's definition is stated in terms of frequencies.

For example, partitions into distinct parts form a partition ideal. Andrews identifies relations between partition ideals which break the set $\mathcal{P}$ into algebraic subclasses.

Definition 5.3. We say two partition ideals $\mathcal{C}, \mathcal{C}^{\prime}$ are equivalent and write $\mathcal{C} \sim \mathcal{C}^{\prime}$ if $\#\{\lambda \in \mathcal{C}:|\lambda|=n\}=\#\left\{\lambda \in \mathcal{C}^{\prime}:|\lambda|=n\right\}$ for all $n \geq 1$.

Andrews carries out the study of equivalences where one subset $\mathcal{C}$ is a partition ideal of "order one" in great detail (see 4] for specifics). These are "nice" subsets of $\mathcal{P}$ including many of interest classically, e.g., partitions into distinct parts form a partition ideal of order one. Sets $\mathcal{P}_{A}, \mathcal{P}_{B}$ as in Theorem 4.5 are also partition ideals of order one. Naturally, then, one wonders if Andrews's theory extends in some way to sets like $\mathcal{P}_{B}(A)$.

A moment's thought convinces one that such sets are not generally partition ideals. However, they do enjoy a tantalizing "quasi-ideal" property: If $a_{i}$ copies (or a multiple thereof) of any part $b_{i}$ are deleted from a partition in $\mathcal{P}_{B}(A)$, the resulting partition is an element of $\mathcal{P}_{B}(A)$ as well.

This feels like a refinement of Definition 5.1 Furthermore, if the $a_{i}$ in the sequence $A$ of distinct terms are rearranged to form a new sequence $A^{\prime}$ (the same terms in a different order), clearly $p_{A^{\prime}}(n)=p_{A}(n)$ even though $\mathcal{P}_{B}\left(A^{\prime}\right) \neq \mathcal{P}_{B}(A) ;$ thus Theorem 4.5 gives

$$
\# \mathcal{P}_{B}(A, n)=\# \mathcal{P}_{B}\left(A^{\prime}, n\right) .
$$

Similarly, noting $B$ is arbitrary in Theorem 4.5 and could be replaced by another subset $B^{\prime} \subseteq \mathbb{N}$ without changing the right side of the second identity, then

$$
\# \mathcal{P}_{B}(A, n)=\# \mathcal{P}_{B^{\prime}}(A, n)
$$


In light of the correspondence between length- $n$ partitions in $\mathcal{P}_{B}(A)$ and size$n$ partitions in $\mathcal{P}_{A}$, equations (5.1) and (5.2) feel similar to partition ideal equivalence in Definition 5.3 .

Moreover, the two-variable generating functions in Section 4 are of a similar shape to Andrews's formulas for "linked partition ideals" in Chapter 8.4 of [4]. Are there maps between these schemes? If subsets of partitions such as $\mathcal{P}_{B}(A)$ are analogous to partition ideals, do there exist closely-related subsets analogous to equivalent ideals in Andrews's theory? Conversely, might cyclic maps like those in Section 3 exist between equivalent partition ideals?

\section{Acknowledgment}

The authors are grateful to the organizers of the Combinatory Analysis 2018 conference and the editors of these proceedings, and to the anonymous referee for many useful comments and references. Furthermore, the second author would like to thank George E. Andrews and Andrew V. Sills for conversations that informed this work.

\section{References}

[1] K. Alladi, G. E. Andrews, and B. Gordon. "Refinements and generalizations of Capparelli's conjecture on partitions." J. Algebra 174 (1995): 636-658.

[2] G. E. Andrews. "Partition identities." Advances in Mathematics 9.1 (1972): 1051.

[3] G. E. Andrews. "Partition ideals of order 1, the Rogers-Ramanujan identities and computers." Groupe d'Etude d'Algebre, 1re Annee 76 (1975).

[4] G. E. Andrews. The Theory of Partitions, Encyclopedia of Mathematics and its Applications, vol. 2, Addison-Wesley, Reading, MA, 1976. Reissued, Cambridge University Press, 1998.

[5] A. Berkovich, and A. K. Uncu. "A new companion to Capparelli's identities." Advances in Applied Mathematics 71 (2015): 125-137.

[6] B. C. Berndt. Number Theory in the Spirit of Ramanujan. American Mathematical Soc., 2006.

[7] J. Dousse. "On partition identities of Capparelli and Primc." arXiv preprint arXiv:1811.02251 (2018).

[8] N. J. Fine. Basic Hypergeometric Series and Applications. Mathematical Surveys and Monographs, 27, American Mathematical Society, 1988.

[9] K. Ono, L. Rolen, and R. Schneider. "Explorations in the theory of partition zeta functions." Exploring the Riemann Zeta Function. Springer, Cham, 2017, 223-264.

[10] R. Schneider. "Partition zeta functions." Research in Number Theory $\mathbf{2 . 1}$ (2016): 9 . 


\author{
Maxwell Schneider \\ Honors Program \\ University of Georgia \\ Athens, Georgia 30602 \\ e-mail: maxwell.schneider@uga.edu \\ Robert Schneider \\ Department of Mathematics \\ University of Georgia \\ Athens, Georgia 30602 \\ e-mail: robert.schneider@uga.edu
}

